\title{
Effects of miR-181a on the biological function of multiple myeloma
}

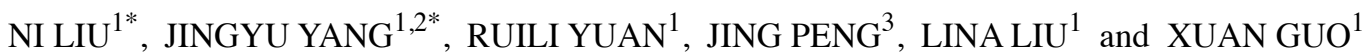 \\ ${ }^{1}$ Department of Clinical Laboratory, The First Affiliated Hospital of Xi'an Jiaotong University, Xi'an, Shaanxi 710061; \\ ${ }^{2}$ Department of Clinical Laboratory, An'kang Hospital of Traditional Chinese Medicine, An'kang, Shaanxi 725000; \\ ${ }^{3}$ Department of Clinical Laboratory, Xi'an Hospital of Traditional Chinese Medicine, Xi'an, Shaanxi 710021, P.R. China
}

Received June 19, 2018; Accepted May 13, 2019

DOI: $10.3892 / o r .2019 .7160$

\begin{abstract}
The present study aimed to investigate the role of miR-181a in multiple myeloma (MM) cell lines. Reverse transcription-quantitative polymerase chain reaction (RT-qPCR) was used to detect the expression of microRNA (miR)-181a. The MM cell line RPMI 8226 stably transduced with miR-181a mimics or inhibitor was established via lentiviral vectors. A Cell Counting Kit- 8 proliferation assay, flow cytometry and a Transwell assay were conducted to assess cell proliferation, cell cycle, apoptosis and invasion. The role of miR-181a in MM tumor formation in vivo was assessed using a SCID Berge xenograft tumor model. The potential target genes of miR-181a were predicted using bioinformatic tools, and the expression of a potential target gene of miR-181a, neuro-oncological ventral antigen-1 (NOVAl) was detected by RT-qPCR . miR-181a was determined to be significantly upregulated in MM cells $(\mathrm{P}<0.001)$. Following silencing of miR-181a via lentiviral-mediated transduction, RPMI 8226 cells exhibited a significant decrease in the number of $S$ phase cells, and proliferative and invasive abilities. In addition, apoptosis was significantly promoted. A total of nine cross-target genes were pre-selected via bioinformatics software, including NOVA1. The results revealed that miR-181a inhibitor suppressed the expression of NOVA1 ( $\mathrm{t}=26.951, \mathrm{P}=0.001)$. In the xenograft tumor model of SCID Berge mice, miR-181a knock down significantly inhibited tumor growth. Conversely, these effects were reversed in response to miR-181 mimics. In summary, miR-181a was determined to be upregulated in MM cells, and may affect the biological function of cancer cells. The underlying mechanism may comprise the regulation of downstream target genes; however, further investigation is required.
\end{abstract}

Correspondence to: Dr Xuan Guo, Department of Clinical Laboratory, The First Affiliated Hospital of Xi'an Jiaotong University, 277 West Yanta Road, Xi'an, Shaanxi 710061, P.R. China E-mail: guox9512@163.com

*Contributed equally

Key words: multiple myeloma, microRNA-181a, biological functions, target gene prediction

\section{Introduction}

Multiple myeloma (MM) is a B-cell malignancy characterized by the abnormal proliferation of plasma cells in the bone marrow (1). For hematological malignancies, the incidence of MM is second only to non-Hodgkin's lymphoma, accounting for $\sim 13 \%$ of hematological malignancies (2). The disease often occurs in the elderly over the age of 60 and with aging of the global population, the incidence of is increasing (3). The treatment of MM mainly comprises chemotherapy, radiotherapy, immunotherapy, autologous stem cell transplantation and targeted therapy (4). Autologous stem cell transplantation can improve patient survival (5); however, as autologous stem cell transplantation is difficult to perform in the elderly and MM is prone to relapse, chemotherapy is administered as the main clinical treatment (4). In recent years, the pathogenesis, development and effective therapies of MM has been extensively studied; however, further investigation is required to identify the mechanism underlying the pathogenesis and develop novel therapeutic strategies for treating this disease (6). Therefore, it is urgent to identify novel targets for the diagnosis and treatment of MM.

MicroRNAs (miRNAs/miRs) are small RNA molecules of $\sim 22$ bases. Studies have reported miRNAs to serve important roles in cellular events, including cell proliferation, differentiation, apoptosis and metabolism (7-10). They also play important regulatory roles in human diseases, such as cancer, blood diseases, cardiovascular diseases and diabetes (11). In 2004, Chen et al (12) demonstrated that miRNAs were involved in the regulation of human hematopoietic system for the first time and the miRNA expression profiles were abnormal in a variety of hematological diseases. The miR-181 family constitutes ubiquitous miRNAs in human cells and are abnormally expressed in a variety of tumors (13). miR-181 has been reported to be expressed in lung cancer, gastric cancer and hematological diseases (14-16). miR-181a of the miR-181 family was determined to be closely related to the regulation of the hematopoietic system $(16,17)$. A total of 12 out of 13 differentially expressed miRNA molecules, including miR-181a, were upregulated in MM (18). We previously reported that miR-181a and miR-20a were highly expressed in serum of patients with MM (19); high expression of miR-181a was proposed as a marker of poor prognosis of MM (20). In addition, our recent study showed that in the serum and bone marrow of patients with MM, miR-181a was highly expressed 
and positively correlated with Durie-Salmon stage, renal function, and $\beta 2$-microglobulin levels (21); however, the role of miR-181a in MM cells and its downstream target genes remain unclear.

The present study aimed to determine the expression profile of miR-181a in MM cells, and analyze the proliferation, cell cycle, apoptosis and invasion of MM cells after transfection of miR-181a in MM cells using lentiviral (LV) transduction. The target genes of miR-181a were predicted by bioinformatics analysis. Animal experiments using nude mice were performed to investigate the effect of miR-181a on MM in vivo.

\section{Materials and methods}

Animals. Female severe combined immunodeficiency (SCID Berge) mice of 3-5 weeks old ( $n=12)$ were purchased from Xi'an Jiaotong University Animal Experimental Center. Mice were housed in standard conditions, under a $12 \mathrm{~h} \mathrm{light/dark}$ cycle, free access to rodent chow and water, room temperature of $18-25^{\circ} \mathrm{C}$ and relative humidity of $40-60 \%$. All experimental procedures involving animals were conducted according to the ethical guidelines of The First Affiliated Hospital of Xi'an Jiaotong University. Ethical approval was received for the use of animals, prior to the start of the study (grant no. XJTULAC2017-782). All efforts were made to minimize animal suffering.

Isolation of human bone marrow lymphocytes. Bone marrow lymphocytes (22) were isolated from 5 patients with benign blood disorders [anemia $(n=3)$ and thrombocytopenia $(n=2)$ ]. Their average age was 55.2 \pm 14.06 years old (age range: 51-73 years). There were 3 female patients (all with anemia) and 2 male patients (all with thrombocytopenia). Prior written and informed consent were obtained from every patient, and the study was approved by the ethics review board of The First Affiliated Hospital of Xi'an Jiaotong University (approval no. XJTU1AF2016LSK-46).

Cell culture. The human MM cell lines RPMI 8226 (a gift from Professor Aili He, Department of Hematology, The Second Affiliated Hospital of Xi'an Jiaotong University) and H929 (a gift from Professor Xiequn Chen, Department of Hematology, Xijing Hospital of Air Force Military Medical University) were cultured in RPMI 1640 medium (HyClone, GE Healthcare Life Sciences) containing 10\% fetal bovine serum (Zhejiang Tianhang Biotechnology Co., Ltd.), and cultured in a humidified incubator containing $5 \% \mathrm{CO}_{2}$ at $37^{\circ} \mathrm{C}$.

Cell transduction and screening. RPMI 8226 cells were seeded into 96-well plates at a concentration of $2 \times 10^{5}$ cells/well. Then, the cells were transfected with LV3-HIV-miR-181a-5p mimics-GFP (LV-miR-181a mimics group), LV3-HIV-miR-181a-5p inhibitor-GFP (LV-miR-181a inhibitor group) and LV3-negative control-GFP (LV-NC), respectively (all from Shanghai GenePharma Co., Ltd.). The sequences were as follows. LV3-miR-181a mimics: 5'-AAC ATTCAACGCTCGGTGAGT-3'; LV3-miR-181a inhibitor: 5'-ACTCACCGACAGCGTTGAATGTT-3'; LV3-NC: 5'-TTC TCCGAACGTGTCACGT-3'. After $48 \mathrm{~h}$, the culture medium was changed. Then, cell culture was continued for another $24 \mathrm{~h}$. Cells were screened with puromycin $(4 \mu \mathrm{g} / \mathrm{ml})$ (Sigma-Aldrich; Merck KGaA). After transduction, cells were observed under a fluorescent microscope (Olympus CKX41; Olympus, Tokyo, Japan). The experiment was repeated three times.

Animal treatment, grouping and sampling. SCID Berge mice were randomly divided into four groups $(n=3)$ : miR-181a mimics, miR-181a inhibitor and two LV-NC groups. RPMI 8226 cells $\left(1 \times 10^{7} /\right.$ cell $)$ transduced with different miRNA-containing vectors were suspended in $100 \mu \mathrm{l}$ PBS and subcutaneously inserted into the back of corresponding SCID Berge mice groups, respectively. The mice were monitored for tumor growth every 3 days. The tumor growth rate was relatively slower in miR-181a inhibitor group than that in miR-181a mimics group. Thus, tumor growth in miR-181a mimics group and its corresponding LV-NC group was observed for 21 days, while that in miR-181a inhibitor group and its corresponding LV-NC group was observed for 33 days. The tumor was measured every 3 days. Tumor volumes were calculated as $1 / 2$ xwidth $(\mathrm{mm}) \mathrm{x}$ length $(\mathrm{mm})$. Tumor bearing mice were sacrificed by cervical dislocation. Then, the tumors were excised and weighed. The expression of neuro-oncological ventral antigen-1 (NOVA1) in tumor tissues was detected by reverse transcription-quantitative polymerase chain reaction (RT-qPCR).

$R T$ - $q P C R$. Total RNAs were extracted from tumor tissues and cells with TRIzol (Thermo Fisher Scientific, Inc.). Then, RNA was reverse transcribed into cDNA using RNA Reverse Transcription Kit (Tiangen Biotech Co., Ltd.) according to the manufacturer's protocols. The miRNA Real-Time PCR Assay kit (Tiangen Biotech Co., Ltd.) was used to detect the expression levels of miR-181a and U6. The PCR procedure for miRNA was $95^{\circ} \mathrm{C}$ for 15 min followed by 45 cycles of $95^{\circ} \mathrm{C}$ for $20 \mathrm{sec}$ and $60^{\circ} \mathrm{C}$ for $34 \mathrm{sec}$. The mRNA Real-Time PCR Assay kit (Tiangen Biotech Co., Ltd.) was used to detect the expression levels of NOVA1 and GAPDH. U6 and GAPDH were used as internal controls. The PCR procedure for mRNA was $95^{\circ} \mathrm{C}$ for $15 \mathrm{~min}$ followed by 45 cycles of $95^{\circ} \mathrm{C}$ for $10 \mathrm{sec}$ and $60^{\circ} \mathrm{C}$ for $30 \mathrm{sec}$. Primers were synthesized by Beijing Dingguo Changsheng Biotechnology Co., Ltd. and the sequences were listed in Table I. The PCR assays were performed using the SuperReal Color Fluorescent Quantitative Premixed Reagent (Tiangen Biotech Co., Ltd.) and an ABI Step one real-time PCR system (Applied Biosystems; Thermo Fisher Scientific, Inc.). The comparative $2^{-\Delta \Delta C q}$ method (23) was used for relative quantification.

Cell Counting Kit-8 (CCK-8) assay. Cell viability was measured using a Cell Counting Kit- 8 assay (Genview). Cells were seeded into 96 -well plates at a concentration of $2 \times 10^{3}$ cells/well for $24 \mathrm{~h}$. After 24, 48 and $72 \mathrm{~h}, 10 \mu \mathrm{l}$ CCK-8 was added in each well. After incubation for $2 \mathrm{~h}$ in $37^{\circ} \mathrm{C} \mathrm{CO}_{2}$ incubator, the absorbance at $450 \mathrm{~nm}$ was measured using an Infinite F50 microplate reader (Tecan Group, Ltd.).

Flow cytometric analysis. Cells were cultured for $24 \mathrm{~h}$, collected by centrifugation at $800 \mathrm{x}$ g for $5 \mathrm{~min}$ at room temperature, and washed with pre-chilled PBS buffer 
Table I. Primers used in the present study.

\begin{tabular}{ll}
\hline Gene & \multicolumn{1}{c}{ Sequence (5'-3') } \\
\hline U6-R & AACGCTTCACGAATTTGCGT \\
U6-F & CTCGCTTCGGCAGCACA \\
miRNA-181a-F & AACATTCAACGCTGTCGGTGAGT \\
miRNA-181a-R & - \\
GAPDH-F & AATGGGCAGCCGTTAGGAAA \\
GAPDH-R & GCGCCCAATACGACCAAATC \\
NOVA1-F & CCCTCCCCAGTGAGAACAAA \\
NOVA1-R & CCGCCATCATGTTTGCAGTT
\end{tabular}

aPrimer was included in the miRNA Real-Time PCR Assay kit. F, forward; R, reverse; miRNA, microRNA; NOVA1, neuro-oncological ventral antigen-1.

(pH 7.2) three times. To investigate the cell cycle, a Cell Cycle Detection Kit (Beyotime Institute of Biotechnology) was used. The cells were fixed with pre-chilled $75 \%$ alcohol at $4^{\circ} \mathrm{C}$ for $24 \mathrm{~h}$ and stained with propidium iodide at $37^{\circ} \mathrm{C}$ in the dark for $30 \mathrm{~min}$. A flow cytometer (BD Biosciences) was then used to analyze cell cycle. To detect apoptosis, cells were resuspended with $195 \mu \mathrm{l}$ assay buffer (included in kit), stained with $5 \mu 1$ Annexin V-phycoerythrin (Beyotime Institute of Biotechnology) in the dark for 10-20 min at room temperature, and then $5 \mu 1$ 7-amino actinomycin D (7-AAD) (Keygentec) in the dark for 5-15 min on ice. Cells were then analyzed by flow cytometry. ModFit LT 5.0 software (Verity Software House, Inc.) was used to analyze the cell cycle, and CellQuest Pro 5.1 (BD Biosciences) software was used to analyze apoptosis. Cells with positive Annexin V and negative 7-AAD were in early apoptosis; cells positive for both Annexin $\mathrm{V}$ and 7-AAD were in late apoptosis.

Transwell invasion assay. Matrigel (5 mg/ml; BD Biosciences) was thawed at $4^{\circ} \mathrm{C}$ overnight and diluted in pre-cooled serum-free medium to a final concentration of $1 \mathrm{mg} / \mathrm{ml}$. Then, $100 \mu \mathrm{l}$ of diluted Matrigel was added to upper chamber (EMD Mililpore) and incubated overnight at $37^{\circ} \mathrm{C}$. Cells were resuspended in serum-free medium at a concentration of $2 \times 10^{5} / \mathrm{ml}$. A total of $750 \mu \mathrm{l}$ of complete medium containing $10 \%$ fetal bovine serum was added to the lower chamber and $200 \mu \mathrm{l}$ of cell suspension was added to the upper chamber. Subsequently, cells were cultured for $18-24 \mathrm{~h}$ at $37^{\circ} \mathrm{C}$. After removing the medium and washing with PBS, cells were fixed with $95 \%$ ethanol at room temperature for $5 \mathrm{~min}$ and permeabilized with $100 \%$ methanol at room temperature for $20 \mathrm{~min}$. After washing, cells were stained with $0.1 \%$ crystal violet at room temperature for $20 \mathrm{~min}$. The non-invading cells were removed using a cotton swab. Invasive cells were counted in 10 randomly selected fields under a fluorescence microscope under bright field (Olympus CKX41; magnification, x200). A hemocytometer was used to count cells under the microscope.

Target gene prediction. Bioinformatics software miRanda (http://miranda.org.uk/), TargetScan5.2 (http://www. targetscan.org/vert_72/) miRDB 5.0 (http://www.mirdb.org/),

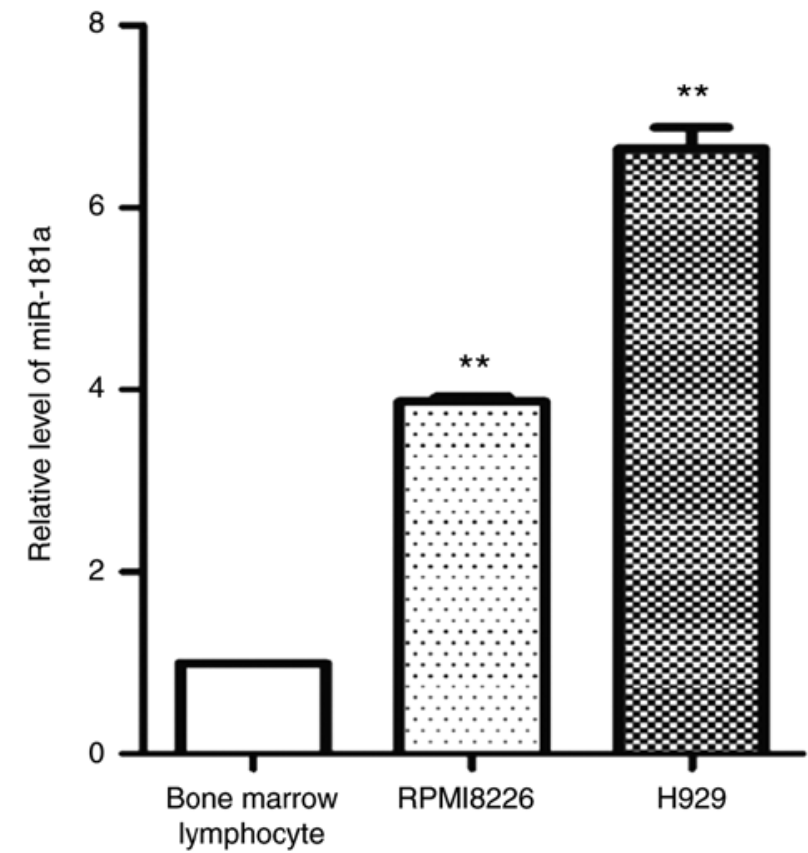

Figure 1. Expression of miR-181a in multiple myeloma cell lines. miR-181a expression was detected via reverse transcription-quantitative polymerase chain reaction. Human bone marrow lymphocytes were used as the control. ${ }^{* * *} \mathrm{P}<0.01$ vs. bone marrow lymphocytes. miR, microRNA.

Pictar (https://pictar.mdc-berlin.de/) and miRTarBase 6.1 (http://mirtarbase.mbc.nctu.edu.tw/php/index.php) were used to predict the target genes of miR-181a.

Statistical analysis. The data was analyzed using SPSS 17.0 (SPSS Inc.) and GraphPad Prism 5.0 (GraphPad Software, Inc.) for Windows ${ }^{\circledast}$. Data from at least three independent experiments were expressed as the mean \pm standard deviation. A Student's t-test or one-way ANOVA followed by a Student-Newman-Keuls test was used when appropriate. $\mathrm{P}<0.05$ was considered to indicate a statistically significant.

\section{Results}

Expression of miR-181a in MM cell lines. RT-qPCR was used to detect the expression of miR-181a in MM cell lines (RPMI 8226 and H929 cells) and bone marrow lymphocytes. The relative expression levels of miR-181a were significantly higher in the MM cell lines (RPMI 8226 and H929 cells) as compared with that in bone marrow lymphocytes (Fig. 1). This result showed that miR-181a was highly expressed in MM cell lines.

In vitro $L V$ transfection efficiency of MM cells. miR-181a mimics, miR-181a inhibitor and LV-NC were transfected into RPMI 8226 cell line by lentivirus. The transduction efficiency was detected and the effects of miR-181a on cell proliferation, cycle, apoptosis and invasion were investigated. A total of $>99 \%$ of dead nontransfected cells were observed on the third day when the concentration of puromycin was $4 \mu \mathrm{g} / \mu \mathrm{l}$ (Fig. 2A). Therefore, $4 \mu \mathrm{g} / \mu \mathrm{l}$ puromycin was used to screen transfected cells and $2 \mu \mathrm{g} / \mu \mathrm{l}$ was used to maintain cultures. Different MOI values for LV transduction conditions 
A
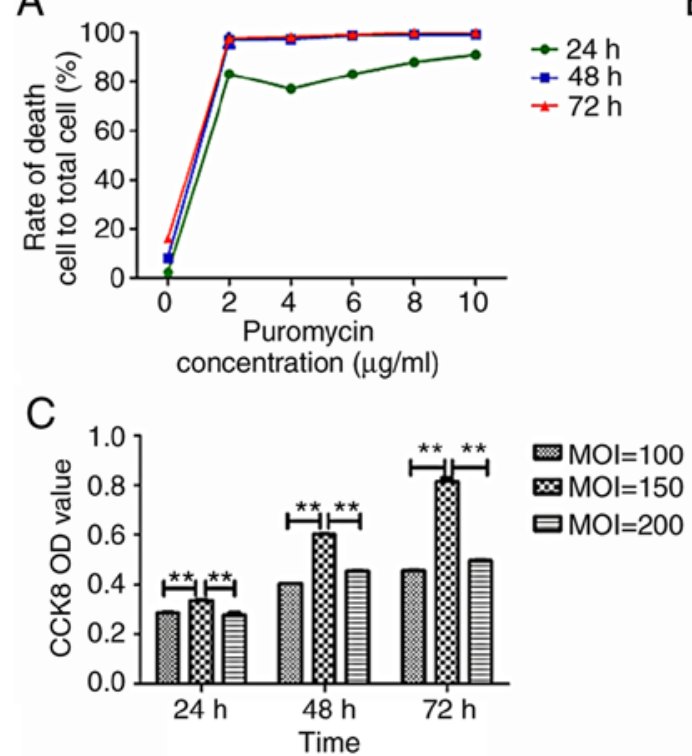

B
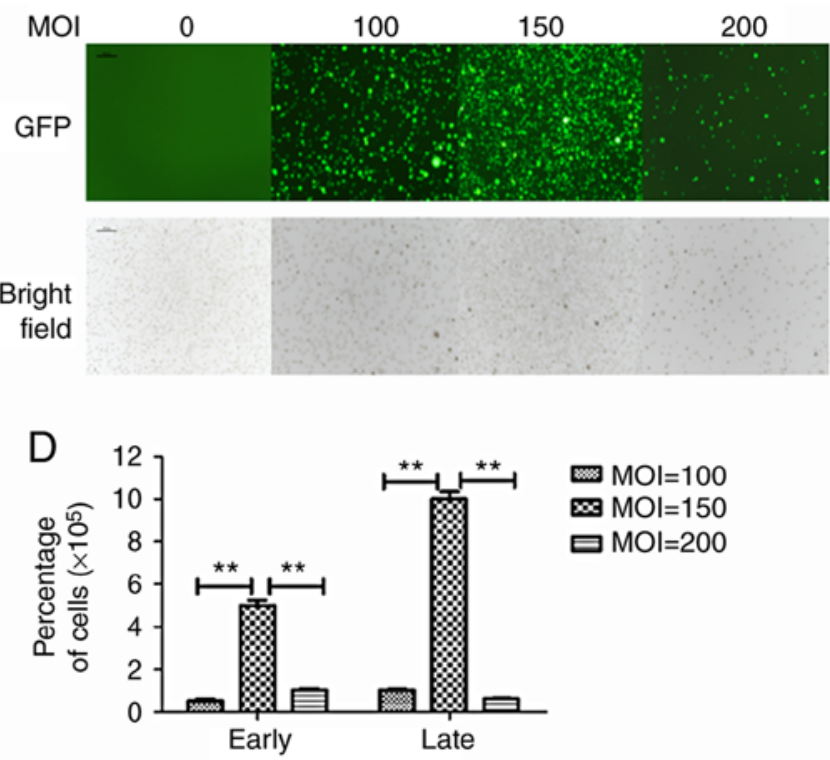

MOI=100

$\bowtie \mathrm{MOI}=150$

घ $\mathrm{MOI}=200$

Figure 2. Establishment of lentiviral transduction of RPMI 8226 cells. (A) Effect of puromycin on RPMI 8226 cell survival. (B) GFP expression of lentiviral transduction with different MOI values after three generations that were screened with puromycin (magnification, $\mathrm{x} 100)$. The (C) cell proliferation and (D) cell number at different time-points following transduction with various MOI values. ${ }^{* *} \mathrm{P}<0.01$. CCK8, Cell Counting Kit-8; GFP, green fluorescent protein; MOI, multiplicity of infection; OD, optical density.

were investigated in three generations. The expression of green fluorescent protein (GFP) under fluorescence microscope was observed (Fig. 2B). The fluorescence intensity was strongest when the MOI value was 150 . Cell proliferation was analyzed via a CCK-8 assay (Fig. 2C) and the cell number (Fig. 2D) also revealed that an MOI of 150 resulted in significantly increased cell viability as compared with MOIs of 100 and 200.

The expression of GFP in the stably transduced cell line after transduction with LV-miR-181a mimics, LV-miR-181a inhibitor and LV-NC was observed under an inverted fluorescence microscope (Fig. 3A and B). The transfection efficiency of the three groups was $>90 \%$. RT-qPCR revealed that miR-181a expression was significantly increased in the miR-181a mimics group compared with the LV-NC group ( $\mathrm{t}=-73.968, \mathrm{P}<0.01$; Fig. $3 \mathrm{C}$ ), and the expression of miR-181a in the miR-181a inhibitor group was significantly downregulated ( $\mathrm{t}=5.068, \mathrm{P}=0.037$; Fig. 3D). These results demonstrated that LV-mediated transduction of cell lines was successfully attained.

Effects of miR-181a on the proliferation of MM cells. A CCK-8 assay was conducted to detect cell proliferation. Compared with the LV-NC group, the proliferation of cells of the miR-181a mimics group significantly increased at 48 and $72 \mathrm{~h}(\mathrm{t}=-3.475$, $\mathrm{P}=0.008$; Fig. 4A), whereas, miR-181a inhibitor cell proliferation was significantly inhibited $(\mathrm{t}=8.098, \mathrm{P}<0.01$; Fig. 4B). This suggests that upregulation of miR-181a expression enhances the proliferative ability of RPMI 8226 cells. Flow cytometry was used to detect the cell cycle status of RPMI 8226 cells. Compared with the LV-NC group (Fig. 5A), the number of cells in G0/G1 phase of the miR-181a mimics group (Fig. 5B) was significantly decreased $(\mathrm{t}=11.529, \mathrm{P}=0.006)$, but increased in $\mathrm{S}$ phase compared with the control $(\mathrm{t}=-27.819$, $\mathrm{P}<0.01$ ) (Fig. 5C). The number of cells in G0/G1 phase of the miR-181a inhibitor group increased significantly $(t=-7.965$, $\mathrm{P}=0.001$ ), but decreased in $\mathrm{S}$ phase compared with the control
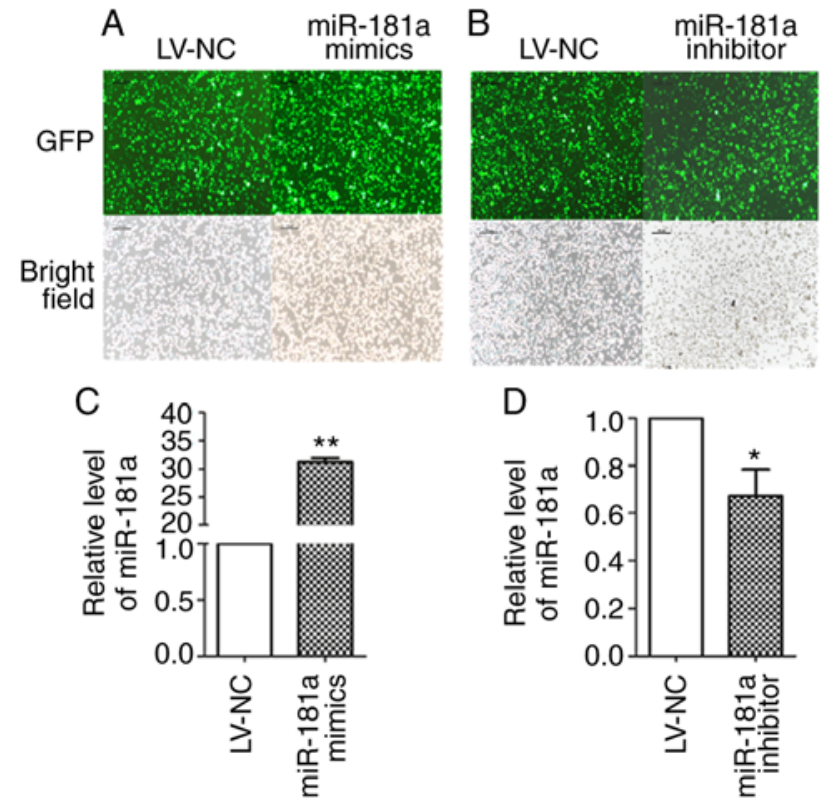

Figure 3. GFP and miR-181a expression following stable LV transduction of cell lines. (A and B) GFP fluorescence following LV transduction of miR-181a mimics and inhibitor. miR-181a expression was determined by reverse transcription-quantitative polymerase chain reaction following LV transduction of (C) miR-181a mimics and (D) miR-181a inhibitor. ${ }^{* *} \mathrm{P}<0.01$ and ${ }^{*} \mathrm{P}<0.05$ vs. LV-NC. GFP, green fluorescent protein; LV, lentiviral; miR, microRNA; NC, negative control.

$(\mathrm{t}=20.610, \mathrm{P}<0.01)$ (Fig. 5D-F). These results indicated that upregulation of miR-181a may induce the progression of the cell cycle to $\mathrm{S}$ phase in RPMI 8226 cells, promoting DNA synthesis and cell proliferation.

Effects of miR-181a transfection on the cell cycle of MM cells. Flow cytometry was also used to detect the apoptosis of each 

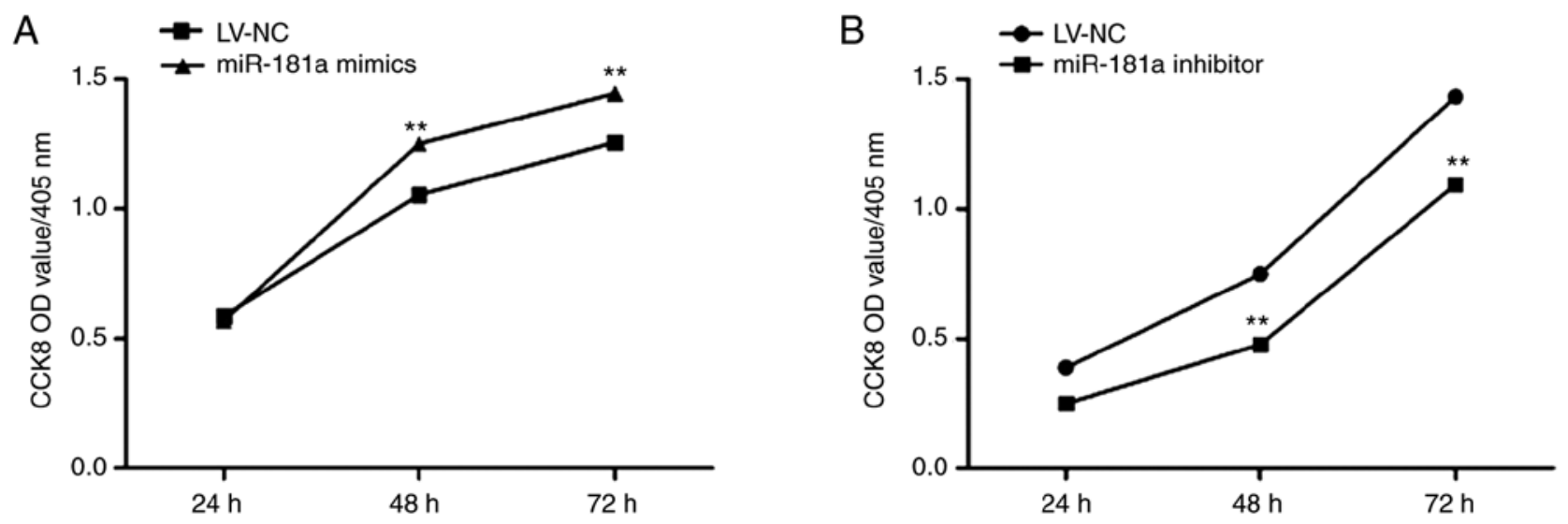

Figure 4. Cell proliferation of cells at different time-points following LV-mediated transduction of miR-181a. A CCK8 assay was used to detect cell proliferation. (A) Cell proliferation after LV-mediated transduction of miR-181a mimics. (B) Cell proliferation after transduction of miR-181a inhibitor. ${ }^{* *} \mathrm{P}<0.01 \mathrm{vs}$. LV-NC. CCK8, Cell Counting Kit-8; LV, lentiviral; miR, microRNA; NC, negative control; OD, optical density.
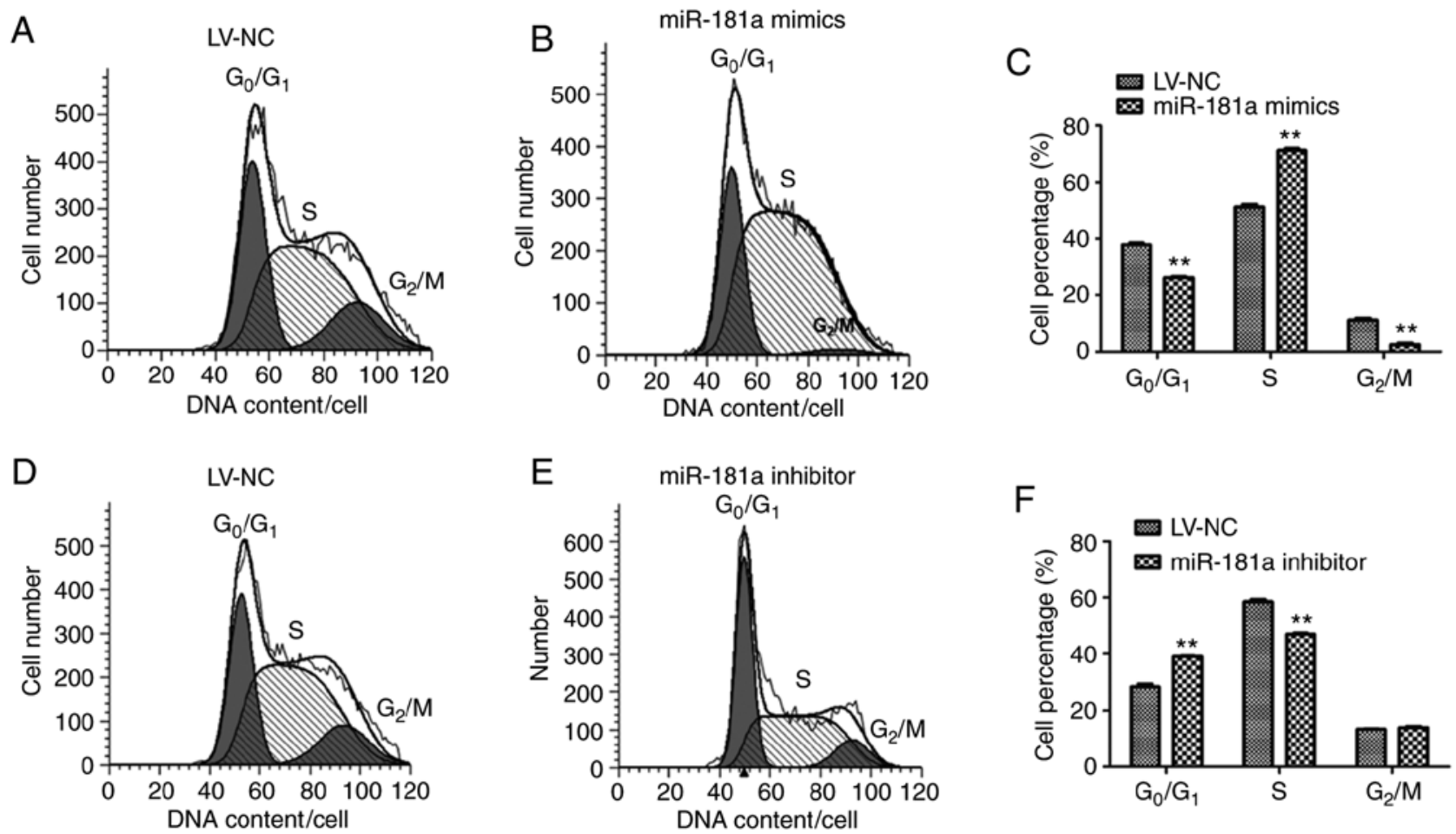

Figure 5. Cell cycle analysis following LV-mediated transduction of miR-181a. The cell cycle after transduction of miR-181a mimics by LV in the (A) LV-NC and (B) miR-181a mimics groups. (C) Proportion of cells after transduction of miR-181a mimics by LV. The cell cycle after transduction of miR-181a inhibitor by LV in the (D) LV-NC and (E) miR-181a inhibitor groups. (F) Proportion of cells after transduction of miR-181a inhibitor by LV. ${ }^{* *} \mathrm{P}<0.01 \mathrm{vs.} \mathrm{LV-NC}$ $\mathrm{LV}$, lentiviral; miR, microRNA; NC, negative control.

group. Compared with the LV-NC group, there was no significant difference in the early apoptotic rate in the miR-181a mimics ( $\mathrm{t}=4.438, \mathrm{P}=0.058$; Fig. 6A-C). While, the early apoptotic rate in the miR-181a inhibitor group (Fig. 6D and E) was significantly higher than that in the LV-NC group $(t=-9.812$, $\mathrm{P}=0.026$; Fig. $6 \mathrm{~F}$ ). This demonstrated that downregulation of miR-181a could enhance the apoptotic rate of RPMI 8226 cells.

Effects of miR-181a transduction on the invasion of MM cells. A Transwell assay was performed to detect cell invasion. As presented in Fig. 7A and B, LV-transduction of miR-181a mimics revealed a significant increase in the number of invaded cells than the LV-NC group ( $\mathrm{t}=-17.978, \mathrm{P}=0.001$; Fig. 7C); however, the number of invaded cells in the inhibitor group was significantly less than that in the LV-NC group ( $\mathrm{t}=14.361$, $\mathrm{P}=0.001$; Fig. 7D-F). These results suggested that upregulation of miR-181a may enhance the invasive ability of RPMI 8226 cells.

Effects of miR-181a on tumor growth in vivo. Xenografts in mice were established using stable transduced cells. Tumor volume was measured every 3 days and the tumors were weighed at the 4th week. The longest tumor diameter for 
A
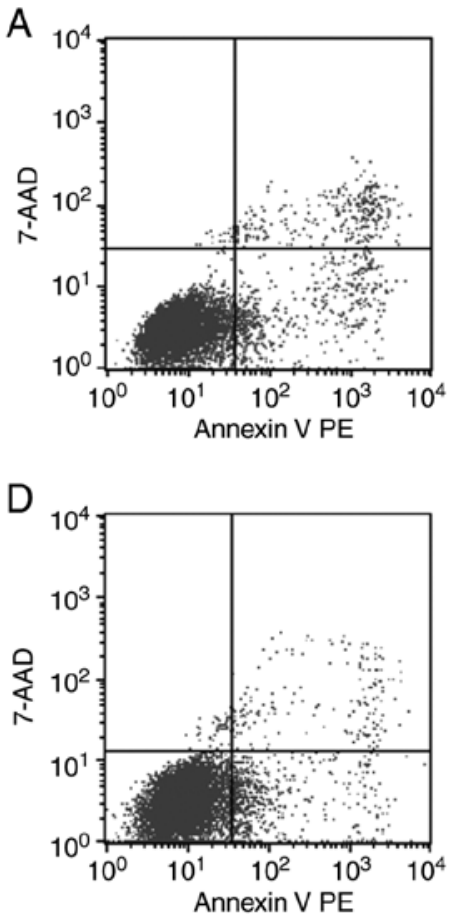

B

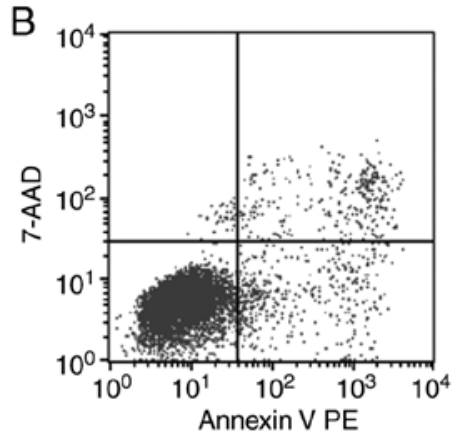

E

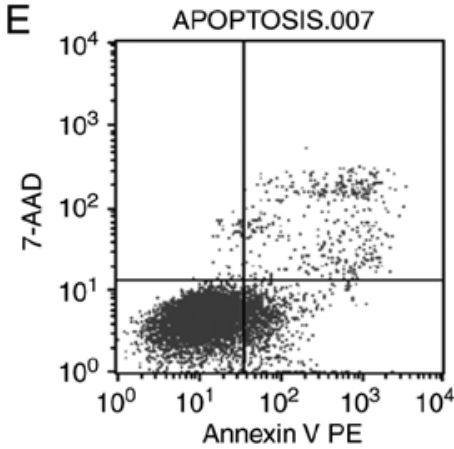

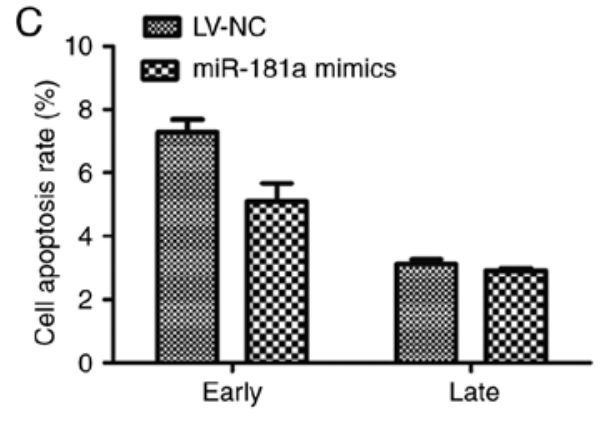

$\mathrm{F}$

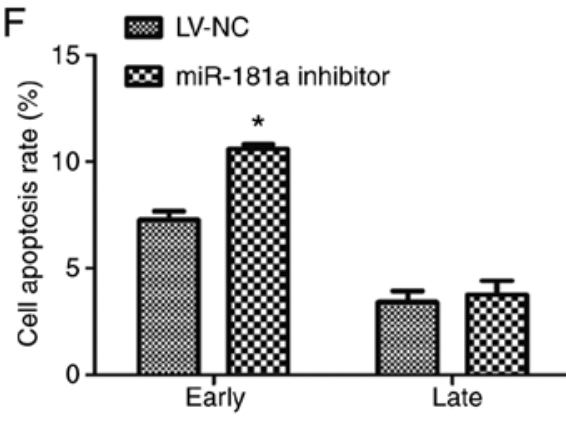

Figure 6. Apoptosis following LV-mediated transduction as detected by flow cytometry. Apoptosis plot after LV-mediated transduction of miR-181a mimics in the (A) LV-NC and (B) miR-181a mimics groups. (C) Proportion of apoptotic cells after LV-mediated transduction of miR-181a mimics. Apoptosis plot after LV-mediated transduction of miR-181a inhibitor in the (D) LV-NC and (E) miR-181a inhibitor groups. (F) Proportion of apoptotic cells after LV-mediated transduction of miR-181a inhibitor. ${ }^{\mathrm{P}}<0.05$ vs. LV-NC. 7-AAD, 7-amino actinomycin; LV, lentiviral; miR, microRNA; NC, negative control; PE, phycoerythrin.

A

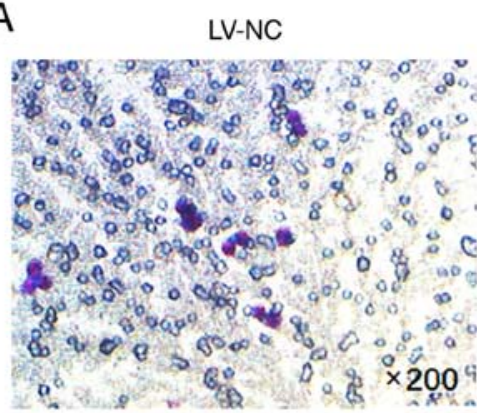

D

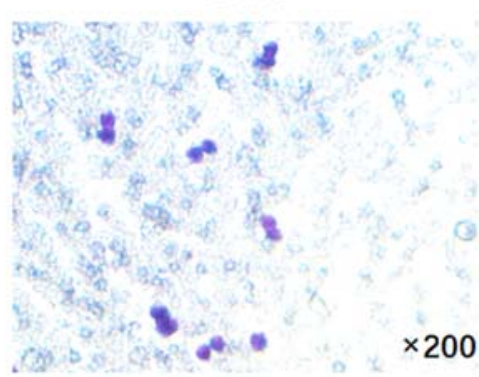

B

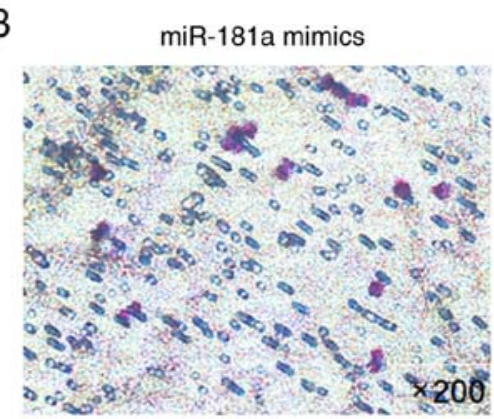

E

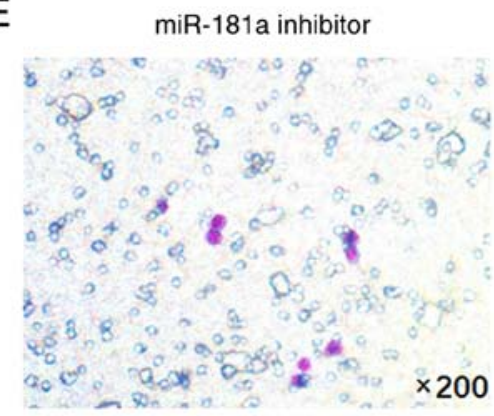

C

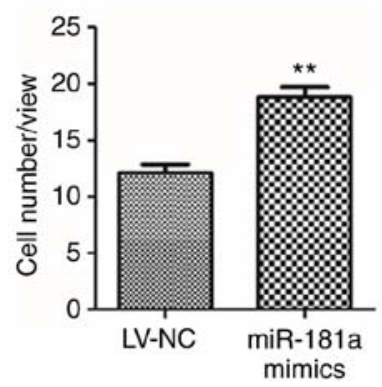

$\mathrm{F}$

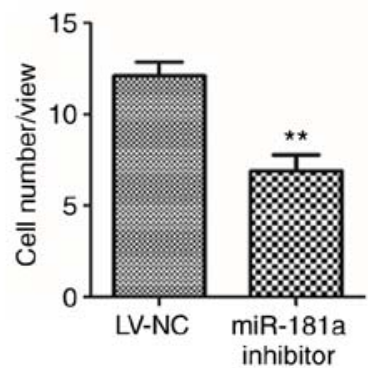

Figure 7. Cell invasion after LV-mediated transduction. Cell invasion after LV-mediated transduction of miR-181a mimics in the (A) LV-NC and (B) miR-181a mimics groups. (C) Statistical analysis following LV-mediated transduction. Cell invasion LV-mediated transduction inhibitor in the (D) LV-NC and (E) miR-181a inhibitor groups. (F) Statistical analysis after LV-mediated transduction of miR-181a inhibitor. Magnification, $\mathrm{x} 200$. ${ }^{* *} \mathrm{P}<0.01 \mathrm{vs}$. LV-NC. LV, lentiviral; miR, microRNA; NC, negative control.

miR-181 mimics group and its corresponding LV-NC group was observed at day 21 , which was $13.59 \times 18.27 \mathrm{~mm}$ and $10.65 \times 12.59 \mathrm{~mm}$, respectively. The longest tumor diameter for the miR-181 inhibitor group and the corresponding LV-NC group was observed at day 33 , which was $11.56 \times 17.97 \mathrm{~mm}$ and 17.89x20.08 mm, respectively. Compared with the LV-NC 

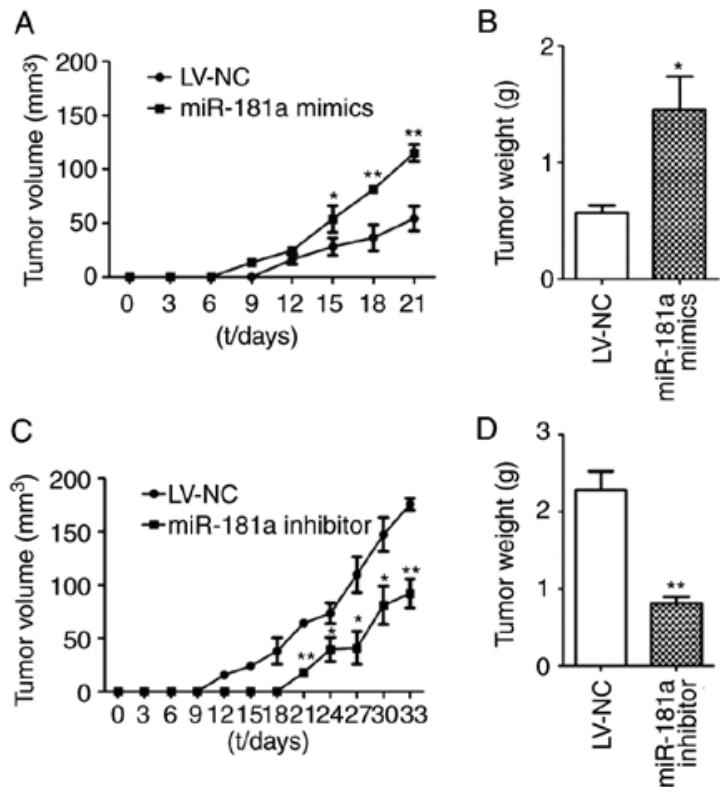

Figure 8. Tumor growth and weight following the establishment of tumor xenografts in mice using stably transduced cells. (A) Tumor growth and (B) weight in the miR-181a mimics group. (C) Tumor growth and (D) weight in miR-181a inhibitor group. ${ }^{* *} \mathrm{P}<0.01$ and ${ }^{*} \mathrm{P}<0.05$ vs. $\mathrm{LV}-\mathrm{NC}$. $\mathrm{LV}$, lentiviral; miR, microRNA; NC, negative control.

group, the tumor volume was significantly larger in the miR-181a mimics group ( $\mathrm{t}=-5.534, \mathrm{P}<0.01$; Fig. $8 \mathrm{~A}$ ); however, the tumor volume was significantly reduced in the miR-181a inhibitor group $(\mathrm{t}=3.249, \mathrm{P}=0.004)$. The tumor weight of the miR-181a mimics group was significantly greater than that of the LV-NC group ( $\mathrm{t}=5.216, \mathrm{P}=0.028$; Fig. $8 \mathrm{~B})$. On the contrary, the tumor weight of the miR-181a inhibitor group was significantly lower than that of the LV-NC group $(t=9.852$, $\mathrm{P}=0.001$; Fig. 8D). These results demonstrated that miR-181a may promote tumor growth in vivo.

Target gene prediction and expression analysis. To study the mechanism of miR-181a on MM, bioinformatics was used to predict the target genes of miR-181a. The number of miR-181a target genes preselected by bioinformatics software (miRanda, TargetScan, miRDB, Pictar, and miRTarBase) was 7,847, 1,367, 887, 457 and 559, respectively. The intersection of the first 100 genes of each database revealed a total of 9 target genes (Table II), including prospero homeobox protein 1, DEAD-box helicase $3 \mathrm{X}$-linked, diphosphoinositol pentakisphosphate kinase 2, NOVA1, interleukin-2, zinc finger protein $780 \mathrm{~A}$, membrane palmitoylated protein 5 , forkhead box P1 and mitogen-activated protein kinase 1.

Studies have reported that NOVA1 can promote cell proliferation in liver cancer, gastric cancer and gliomas $(20,24,25)$. Therefore, the mRNA expression levels of NOVAl in transfected MM cells was detected by RT-qPCR. Compared with the LV-NC group, the miR-181a mimics group (Fig. 9A) showed no significant change $(\mathrm{t}=9.551, \mathrm{P}=0.011)$, while the NOVAl mRNA expression in the miR-181a inhibitor group (Fig. 9B) was significantly inhibited.

RT-qPCR was used to detect the expression of NOVAl at the mRNA level (Fig. 10A and B) in tumor xenograft samples. LV-mediated transduction of miR-181a mimics exhibited no significant effects on the expression of NOVA1, while miR-181a inhibitor significantly downregulated the expression of NOVAl compared with the LV-NC group. The results were consistent with those of the in vitro cell experiments.

\section{Discussion}

MM is a hematological malignancy that accounts for $\sim 13 \%$ of hematological malignancies (2). In recent years, the incidence of MM has increased annually with aging of the population worldwide (3). The pathogenesis of MM is complex; few symptoms are observed during the early stages of disease and relapse frequently occurs. Clinically, this disease cannot be cured (6). Therefore, it is necessary to thoroughly study the pathogenesis of $\mathrm{MM}$ in order to reveal its molecular mechanism, and identify novel targets for the diagnosis and treatment of MM.

miRNAs are small RNA molecules of 22 bases in length that regulate gene expression at the post-transcriptional level. The mechanism of its regulation involves the inhibition of translation or the degradation of mRNA by binding to the mRNA complementary sequence of the target gene, thereby inhibiting the expression of the target gene (26). miRNA regulation serves important regulatory roles in cell proliferation, circulation, migration and invasion (27-29); dysregulated miRNAs have been associated with the occurrence and development of cancer (30-32).

A study has reported that miR-181a is highly expressed in breast and pancreatic cancers, but is downregulated in gliomas and invasive lymphocytes (33). In non-small cell lung cancer (33), human breast and colon cancer (34), and cervical cancer cells (34), miR-181a serves roles in cell proliferation, invasion and migration, and promotes colorectal cancer cell proliferation and drug resistance via the $\mathrm{Wnt} / \beta$-catenin signaling pathways (35). Furthermore, miR-181a promotes gastric cancer cell proliferation, invasion, and inhibition of apoptosis by downregulating myotubularin related protein $3(30,32)$. These findings suggest that miR-181a plays an important role in the development of tumors. To explore the association between miR-181a and MM, RT-qPCR was conducted to detect miR-181a expression in different MM cell lines. We reported that miR-181a was upregulated in MM cell lines. We have not identified methods for the extraction of normal plasmacytes in the literature; MM is a B cell clone proliferative disease. Thus, normal lymphocytes, but not normal plasmacytes, were used as control cells in the present study.

To investigate the effects of miR-181a on MM in vivo, tumor growth was observed every 3 days in an animal model of xenograft tumors. The results showed that tumor growth and weight of the miR-181a mimics group were significantly promoted than that of the control group. The tumor growth of miR-181a inhibitor group was inhibited, and the tumor weight was significantly less than the control group. Thus, these results indicated that miR-181a could promote the growth of MM tumors. This is consistent with our findings that miR-181a promotes MM cell proliferation in vitro. miR-181a was reported to promote colorectal cancer cell proliferation via the Wnt/ $\beta$-catenin signaling pathway (33).

To further observe the effects of miR-181a on the biological function of MM, the expression of miR-181a was upregulated 
Table II. Target genes of miR-181a as predicted with bioinformatics tools.

\begin{tabular}{|c|c|c|c|c|c|c|c|}
\hline $\mathrm{miR}$ & Gene & miRanda & targetScan & miRDB & MiRTarBase & Pictar & SUM \\
\hline Hsa-miR-181a & PROX1 & & & 1 & 1 & & 2 \\
\hline Hsa-miR-181a & DDX3X & 1 & & 1 & & & 2 \\
\hline Hsa-miR-181a & PPIP5K2 & 1 & & 1 & & & 2 \\
\hline Hsa-miR-181a & NOVA1 & 1 & & 1 & & 1 & 3 \\
\hline Hsa-miR-181a & IL-2 & 1 & 1 & 1 & & & 3 \\
\hline Hsa-miR-181a & ZNF780A & 1 & 1 & & & & 2 \\
\hline Hsa-miR-181a & MPP5 & 1 & & & & 1 & 2 \\
\hline Hsa-miR-181a & FOXP1 & 1 & 1 & & & & 2 \\
\hline Hsa-miR-181a & MAPK1 & 1 & 1 & & 1 & & 3 \\
\hline
\end{tabular}

DDX3X, DEAD-box helicase 3 X-linked; FOXP1, forkhead box P1; Hsa, homo sapiens; IL-2, interleukin-2; miR, microRNA; MAPK1, mitogen-activated protein kinase 1; MPP5, membrane palmitoylated protein 5; PPIP5K2, diphosphoinositol pentakisphosphate kinase 2; PROX1, prospero homeobox protein 1; ZNF780A, zinc finger protein 780A.
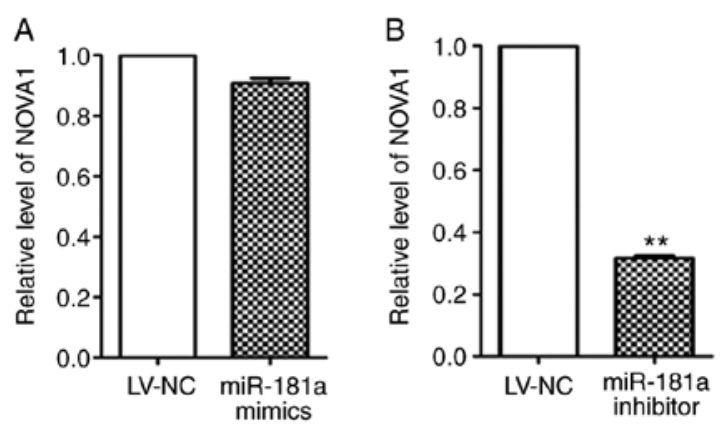

Figure 9. NOVA1 expression after LV-mediated transduction of cells. (A) mRNA level of NOVA1 in miR-181a mimics stable cell lines. (B) mRNA level of NOVA1 in miR-181a inhibitor stable cell lines. LV, lentiviral; miR, microRNA; NC, negative control; NOVA1, neuro-oncological ventral antigen-1. ${ }^{* *} \mathrm{P}<0.01$, vs. LV-NC.

and downregulated via lentiviral-mediated cell transduction. The effects of miR-181a expression on cell proliferation, cell cycle, apoptosis and invasion were observed by CCK-8, flow cytometry and Transwell assays. The results revealed that cell proliferation was inhibited, the number of cells in $\mathrm{S}$ phase of the cell cycle was significantly reduced, and the rate of early apoptosis increased after downregulation of miR-181a. Cell content in S phase can reflect the proliferative ability of the tumor and can also be used to determine the degree of malignancy (36). Generally, the higher the number of cells in S phase, the greater the degree of malignancy, which has been associated with the poor prognosis of patients (36). These results suggest that miR-181a may be used as a marker to predict the prognosis of patients. Cell invasion experiments showed that miR-181a significantly promoted the invasion of tumor cells. After inhibiting the expression of miR-181a, the early apoptosis rate of cells increased. This may benefit the development of therapeutic strategies for the treatment of MM.

To further explore the molecular mechanisms underlying the effects of miR-181a on MM cell cycle, proliferation, apoptosis, and invasion, six target genes were predicted using various bioinformatics software. The expression of NOVAl
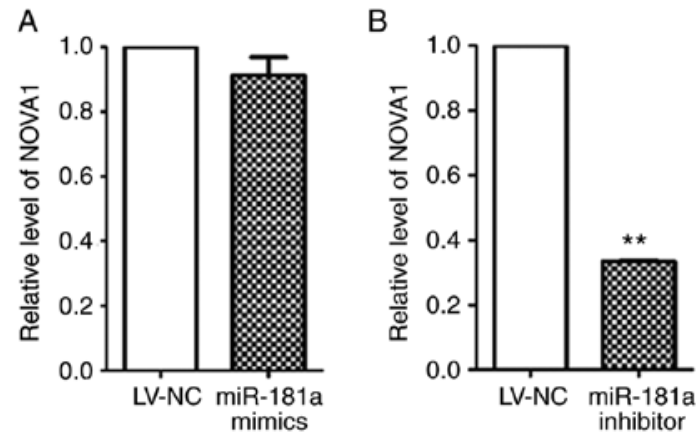

Figure 10. NOVA1 expression in tumor xenografts transduced via LV vectors (A) mRNA level of NOVA1 in tumor xenografts transduced with miR-181a mimics. (B) mRNA level of NOVA1 in tumor xenografts transduced with miR-181a inhibitor. LV, lentiviral; miR, microRNA; NC, negative control; NOVA1, neuro-oncological ventral antigen-1. ${ }^{* *} \mathrm{P}<0.01$, vs. LV-NC.

was verified by RT-qPCR. Our results revealed that NOVAI mRNA expression was reduced in the miR-181a inhibitor group. This opposes the known functions of miRNAs, as the mRNA expression should be induced providing $B C L-2$ and NOVAl were targets of miR-181a. Several studies have reported that miRNAs can activate downstream gene expression by binding to promoters to initiate gene transcription (37-39), which may explain our experimental results. It is also possible that miR-181a targets and regulates upstream of NOVAl, thus affecting its expression; the underlying mechanism requires further investigation.

There are some limitations to the present study. Our previous study (19) revealed that miR-181a and miR-20a were highly expressed in the serum of patients with MM, whereas the present study reported that miR-181a was upregulated in MM cell lines. These results suggest that miR-181a may be involved in the pathogenesis of myeloma; however, we failed to isolate primary myeloma cells from patients with MM. Further studies with primary myeloma cells are required to verify the role of miR-181a in myeloma.

In conclusion, this research has comprehensively studied the biological effects of miR-181a on MM in vitro and in vivo. miR-181a was upregulated in MM cell lines, and promoted 
cell proliferation, increased the ratio of $S$ phase cells, inhibited apoptosis and promoted tumor growth. As a tumor-promoting factor, miR-181a may play an important role in vitro in cell lines and in vivo in animal xenograft models. Predicting the target genes of miR-181a may provide a basis for further exploration into the mechanism underlying the effects of miR-181a in MM.

\section{Acknowledgements}

Not applicable.

\section{Funding}

This work was supported by the Ministry of Science and Technology of Shaanxi (grant no. S2016YFKW0040).

\section{Availability of data and materials}

All data generated or analyzed during this study are included in this published article.

\section{Authors' contributions}

NL performed the experiments (including RT-qPCR, CCK-8, flow cytometry, Transwell assays and animal experiments), analyzed the data and wrote the manuscript. JY performed the animal experiments and analyzed the data. RY participated in RT-qPCR and flow cytometry analysis. JP and LL were involved in the CCK- 8 and Transwell assays. XG conceived the idea, designed the study and helped to revise the manuscript. All authors read and approved the final manuscript.

\section{Ethics approval and consent to participate}

Prior written and informed consent were obtained from every patient, and the study was approved by the ethics review board of The First Affiliated Hospital of Xi'an Jiaotong University (approval no. XJTU1AF2016LSK-46). All experimental procedures involving animals were conducted according to the ethical guidelines of The First Affiliated Hospital of Xi'an Jiaotong University. Ethical approval was received for the use of animals, prior to the start of the study (grant no. XJTULAC2017-782).

\section{Patient consent for publication}

Not applicable.

\section{Competing interests}

The authors declare that they have no competing interests.

\section{References}

1. Li Y, Bai O, Liu C, Du Z, Wang X, Wang G and Li W: Association between hepatitis $B$ virus infection and risk of multiple myeloma: A systematic review and meta-analysis. Intern Med J 46: 307-314, 2016.

2. Palumbo A and Anderson K: Multiple myeloma. N Engl J Med 364: 1046-1060, 2011.
3. Dürner J, Reinecker $\mathrm{H}$ and Csef $\mathrm{H}$ : Individual quality of life in patients with multiple myeloma. Springerplus 2: 397, 2013.

4. Nooka AK, Kastritis E, Dimopoulos MA and Lonial S: Treatment options for relapsed and refractory multiple myeloma. Blood 125: 3085-3099, 2015.

5. Patriarca F, Fanin R, Silvestri F, Damiani D and Baccarani M Autologous stem cell transplantation in multiple myeloma: A single center experience. Haematologica 83: 477-479, 1998.

6. Zhao Z, Ma X, Sung D, Li M, Kosti A, Lin G, Chen Y, Pertsemlidis A, Hsiao TH and Du L: microRNA-449a functions as a tumor suppressor in neuroblastoma through inducing cell differentiation and cell cycle arrest. RNA Biol 12: 538-554, 2015.

7. Ambros V: The functions of animal microRNAs. Nature 431: 350-355, 2004

8. Lu J, Getz G, Miska EA, Alvarez-Saavedra E, Lamb J, Peck D, Sweet-Cordero A, Ebert BL, Mak RH, Ferrando AA, et al: MicroRNA expression profiles classify human cancers. Nature 435: 834-838, 2005.

9. Michael MZ, O' Connor SM, van Holst Pellekaan NG, Young GP and James RJ: Reduced accumulation of specific microRNAs in colorectal neoplasia. Mol Cancer Res 1: 882-891, 2003.

10. Tutar Y: miRNA and cancer; Computational and experimental approaches. Curr Pharm Biotechnol 15: 429, 2014.

11. Deb B, Uddin A and Chakraborty S: miRNAs and ovarian cancer: An overview. J Cell Physiol 233: 3846-3854, 2018.

12. Chen CZ, Li L, Lodish HF and Bartel DP: MicroRNAs modulate hematopoietic lineage differentiation. Science 303: 83-86, 2004.

13. Liu G, Li Y and Gao XG: microRNA-181a is upregulated in human atherosclerosis plaques and involves in the oxidative stress-induced endothelial cell dysfunction through direct targeting Bcl-2. Eur Rev Med Pharmacol Sci 20: 3092-3100, 2016.

14. Cao Y, Zhao D, Li P, Wang L, Qiao B, Qin X, Li L and Wang Y: MicroRNA-181a-5p impedes IL-17-induced nonsmall cell lung cancer proliferation and migration through targeting VCAM-1. Cell Physiol Biochem 42: 346-356, 2017.

15. Liu Z, Sun F, Hong Y, Liu Y, Fen M, Yin K, Ge X, Wang F, Chen $X$ and Guan W: MEG2 is regulated by miR-181a-5p and functions as a tumour suppressor gene to suppress the proliferation and migration of gastric cancer cells. Mol Cancer 16: 133, 2017.

16. Su R, Lin HS, Zhang XH, Yin XL, Ning HM, Liu B, Zhai PF, Gong JN, Shen C, Song L, et al: MiR-181 family: Regulators of myeloid differentiation and acute myeloid leukemia as well as potential therapeutic targets. Oncogene 34: 3226-3239, 2015.

17. Lyu X, Li J, Yun X, Huang R, Deng X, Wang Y, Chen Y and Xiao G: miR-181a-5p, an inducer of Wnt-signaling, facilitates cell proliferation in acute lymphoblastic leukemia. Oncol Rep 37: 1469-1476, 2017.

18. Xiang T, Hu AX, Sun P, Liu G, Liu G and Xiao Y: Identification of four potential predicting miRNA biomarkers for multiple myeloma from published datasets. PeerJ 5: e2831, 2017.

19. Peng J, Thakur A, Zhang S, Dong Y, Wang X, Yuan R, Zhang K and Guo X: Expressions of miR-181a and miR-20a in RPMI 8226 cell line and their potential as biomarkers for multiple myeloma. Tumour Biol 36: 8545-8552, 2015.

20. Shen B, Zhang Y, Yu S, Yuan Y, Zhong Y, Lu J and Feng J: MicroRNA-339, an epigenetic modulating target is involved in human gastric carcinogenesis through targeting NOVA1. FEBS Lett 589: 3205-3211, 2015.

21. Yuan R, Liu N, Yang J, Peng J, Liu L and Guo X: The expression and role of miR-181a in multiple myeloma. Medicine (Baltimore) 97: e12081, 2018.

22. Tassone G and Fidler SJ: Separation and cryopreservation of lymphocytes from spleen and lymph node. Methods Mol Biol 882: 351-357, 2012.

23. Livak KJ and Schmittgen TD: Analysis of relative gene expression data using real-time quantitative PCR and the 2(-Delta Delta $\mathrm{C}(\mathrm{T})$ ) method. Methods 25: 402-408, 2001.

24. Xin Y, Li Z, Zheng H, Ho J, Chan MTV and Wu WKK: Neuro-oncological ventral antigen 1 (NOVA1): Implications in neurological diseases and cancers. Cell Prolif 50: 2017.

25. Zhang YA, Liu HN, Zhu JM, Zhang DY, Shen XZ and Liu TT: RNA binding protein Nova1 promotes tumor growth in vivo and its potential mechanism as an oncogene may due to its interaction

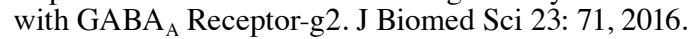

26. Zhou $\mathrm{H}$ and $\mathrm{Wu} \mathrm{L}$ : The development and function of dendritic cell populations and their regulation by miRNAs. Protein Cell 8: 501-513, 2017. 
27. Bartel DP: MicroRNAs: Genomics, biogenesis, mechanism, and function. Cell 116: 281-297, 2004.

28. Zhang Y, Yang P and Wang XF: Microenvironmental regulation of cancer metastasis by miRNAs. Trends Cell Biol 24: 153-160, 2014.

29. Shin VY and Chu KM: MiRNA as potential biomarkers and therapeutic targets for gastric cancer. World J Gastroenterol 20: 10432-10439, 2014.

30. Calin GA and Croce CM: MicroRNA signatures in human cancers. Nat Rev Cancer 6: 857-866, 2006.

31. Manikandan J, Aarthi JJ, Kumar SD and Pushparaj PN Oncomirs: The potential role of non-coding microRNAs in understanding cancer. Bioinformation 2: 330-334, 2008.

32. Miska EA: How microRNAs control cell division, differentiation and death. Curr Opin Genet Dev 15: 563-568, 2005.

33. Yu W, Li L, Zheng F, Yang W, Zhao S, Tian C, Yin W, Chen Y, Guo W, Zou L and Deng W: $\beta$-catenin cooperates with CREB binding protein to promote the growth of tumor cells. Cell Physiol Biochem 44: 467-478, 2017.

34. Yang M,Zhai X, Ge T, Yang C and Lou G: miR-181a-5p promotes proliferation and invasion and inhibits apoptosis of cervical cancer cells via regulating inositol polyphosphate-5-phosphatase A (INPP5A). Oncol Res 26: 703-712, 2018.
35. Han P, Li JW, Zhang BM, Lv JC, Li YM, Gu XY, Yu ZW, Jia YH, Bai XF,Li L, et al: The lncRNA CRNDE promotes colorectal cancer cell proliferation and chemoresistance via miR-181a-5p-mediated regulation of Wnt/ $\beta$-catenin signaling. Mol Cancer 16: 9, 2017.

36. Arora $S$ and Tandon S: Achyranthes aspera root extracts induce human colon cancer cell (COLO-205) death by triggering the mitochondrial apoptosis pathway and $\mathrm{S}$ phase cell cycle arrest. ScientificWorldJournal 2014: 129697, 2014.

37. Janowski BA, Younger ST, Hardy DB, Ram R, Huffman KE and Corey DR: Activating gene expression in mammalian cells with promoter-targeted duplex RNAs. Nat Chem Biol 3: 166-173, 2007.

38. Li LC, Okino ST, Zhao H, PookotD, Place RF, Urakami S, Enokida H and Dahiya R: Small dsRNAs induce transcriptional activation in human cells. Proc Natl Acad Sci USA 103: 17337-17342, 2006.

39. Place RF, Li LC, Pookot D, Noonan EJ and Dahiya R: MicroRNA-373 induces expression of genes with complementary promoter sequences. Proc Natl Acad Sci USA 105: 1608-1613, 2008. 